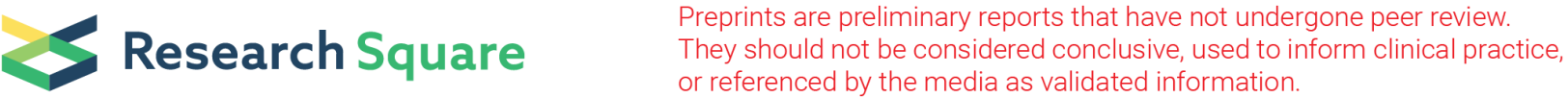

\section{Prevalence of school absenteeism during menstruation and associated factors in sub- Saharan Africa: A systematic review and meta- analysis protocol}

\section{Amerti Gelo ( $\square$ amuayu26@gmail.com )}

Ambo University College of Medicine and Public Health

\section{Melake Damena}

Haramaya University College of Health Sciences: Haramaya University College of Health and Medical Sciences

\section{Ayele Geleto}

Haramaya University College of Health Sciences: Haramaya University College of Health and Medical Sciences

\section{Abera Kenay}

Haramaya University College of Health Sciences: Haramaya University College of Health and Medical

Sciences

\section{Protocol}

Keywords: School absenteeism, Menstruation, Female students, Sub-Sahara Africa

Posted Date: April 13th, 2021

DOI: https://doi.org/10.21203/rs.3.rs-415160/v1

License: (c) (i) This work is licensed under a Creative Commons Attribution 4.0 International License. Read Full License 


\section{Abstract}

Background: Although menstruation is a normal physiological process, it could affect several females' daily activities. In most low resource settings, where menstrual hygiene management is poor and several schools have no water supply and separate toilet for female students, most school girls miss classes during menstruation. With the emergence of studies on menstrual hygiene management, it is evident that pooling several pocket studies is essential to draw lessons about effects of menstruation on female students' class participation. Therefore, the aim of this review is to systematically assess the prevalence of school absenteeism and associated factors during menstruation among high school students in subSaharan Africa.

Methods: The protocol for this systematic review was registered in PROSPERO (ID CRD42020223447). Electronic databases such as MEDLINE, CINAHL, Embase, Web of Science, Scopus and Google Scholar will be searched for published articles using appropriate key terms. The identified articles will be assessed for meeting the eligibility criteria such as being published in English, conducted in sub-Saharan Africa, contain information on school absenteeism, and conducted in schools. Two reviewers will independently screen the identified articles using titles and abstracts followed by full text review of all potential articles. The quality of the included papers will be assessed using the adapted NewcastleOttawa Scale for cross-sectional studies. The Preferred Reporting Items for Systematic Reviews and Meta-Analyses (PRISMA) checklist will be employed to present the findings. Quantitative findings will be pooled using random effect meta-analysis model while the qualitative findings will be presented using narrative synthesis.

Discussion: Analysis of existing evidence of school absenteeism during menstruation is essential for designing appropriate interventions in school and beyond. This review will present credible information that would help in designing evidence-based menstrual hygiene management for improving school attendance during menstruation in sub-Saharan Africa where more than half of female students miss classes during menstruation.

Systematic review registration: PROSPERO CRD42020223447

\section{Background}

Female adolescence has been recognized as a special period that requires special attention as it is marked by several physiological changes. Menarche is a unique change in women that is characterized with initiation of menstruation (1). Although menstruation is a normal physiological process among reproductive age women, it is also linked with several misconceptions and malpractices-especially in low resource settings (2). During menarche, girls who lack prior information about menstruation experience different feelings such as fear, shame and guilt (3). In addition, initiation of menstruation may limit their daily activities resulting in missing schools due to lack of proper menstruation hygiene management (MHM) or lack of sanitary materials in schools (4). 
In low and middle income countries, poor MHM in school is a major problem for female students to attend their school (5). Missing schools during menstruation among female students is mainly attributed to lack of water, sanitation and hygiene (WASH) facilities in schools, inadequate puberty education, and lack of menstruation hygiene items (6). Due to inadequate WASH facilities in school, female students feel humiliation of menstrual blood leakage and bad body odour, and therefore may prefer to remain at home (7). Moreover, poor MHM has also been associated with serious health problems like reproductive tract and urinary tract infections that might end in missing classes $(8,9)$.

Dysmenorrhea is another most important causes of school absenteeism among female students during menstruation (10). Dysmenorrhea refers to the pain associated with menstruation and is the most common gynaecologic complaints in adolescence girls (11-13) that usually starts during menstruation (14). Dysmenorrhea, compounded with lack of prior information about menstruation (11) and cultural practices that impose restrictions on girls' activities during menstruation moments (15), is the leading causes of school absenteeism among menstruating students (16). Dysmenorrhea manifested in such a way that menstruating women suffer either abdominal pain, backache, stomach cramp, waist pain, thigh pain or the combination of these symptoms (11-13).

Appropriate MHM in school is crucial for physical and mental health, education, and dignity of school girls (17). In low resource settings, where more than half of the schools lack sufficient and separate toilets for girls (18), female students are challenged to maintain their menstrual hygiene while they are in school. Female students whose menstruation is not well managed, feel discomfort and shame sitting beside male students in the class (19). Sometimes female students are obliged to carry the used absorbent materials back home due to lack of appropriate absorbent disposal system in most schools (20).

While PMHM affected school attendance for the majority of female students in low- and middle-income countries (21-23), this problem is more rooted in sub-Saharan Africa. For example, a study conducted in Lebanon showed that $41.4 \%$ of female students missed classes (24) while in India, about $40 \%$ of school girls missed classes during menstruation . In sub-Sahara African countries, where $90 \%$ of schools lack separate sanitary facility for females (25), one in ten school girl couldn't attend the school during menstruation (26). For instance, in Uganda, 19.7\% female students reported that they missed at least 1 day of school during their most recent menstrual period (27) whereas more than half of female students missed classes during their menstruation in Ethiopia (19).

Despite significant effects of menstruation on female daily activities, much attention is not given to this problem as influences of menstruation on female students' education is neglected. In sub-Saharan Africa, evidence about school absenteeism during menstruation and its associated factors was scarce. Given that several pocket studies are emerging, there is a need to systematically address these studies to generate accurate evidence to draw lessons about the problem and its mitigation. Findings of this review will become an input for organizations working on adolescent health in solving problems pertaining to menstruation, hence has paramount advantage for female students living in sub-Saharan Africa. 
Evidence about the effect of menstruation on school attendance is demanding for policy and practice, especially in sub-Saharan Africa where there is scarcity of such information. Therefore, this systematic review and meta-analysis is designed to identify level of school absenteeism among adolescent girls during menstruation and determinant factors in sub-Saharan Africa.

\section{Methods}

\section{Data sources}

Articles published on school absenteeism during menstruation among female students in sub-Saharan Africa will be systematically searched from several online electronic databases. The protocol for this review is registered in the International Prospective Register of Systematic Reviews (PROSPERO, CRD 42020223447). The Preferred Reporting Items for Systematic Reviews and Meta-Analyses (PRISMA) checklist (28) will be employed to summarise and present the findings. The Meta-analysis Of Observational Studies in Epidemiology (MOOSE) guideline will be employed to conduct the meta-analysis and to report the findings (29) and qualitative findings will be organized and presented using narrative synthesis.

\section{Search strategy}

Published journal articles will be searched online in MEDLINE, CINAHL, Embase, Web of Science, Scopus and Google Scholar electronic databases using appropriate keywords. A comprehensive search strategy and search terms that are developed using appropriate Boolean operators will be used to locate suitable articles. The key search terms and phrases used to locate appropriate articles include 'menstruation or menstrual or menses or menstruation period or menstrual cycle' AND "hygiene OR sanitation OR sanitary' AND 'absence OR missing OR dropout OR school attendance OR absenteeism' AND 'school or high school or secondary school' AND 'female students or girl students or female pupils' AND 'Sub-Saharan Africa OR Africa south of the Sahara'. Detail of sample search terms for MEDLINE database is presented as supplementary file (Supplementary file 1). In addition, 'grey literature' such as reports from UNICEF about school sanitation and water supply, and the reference lists of eligible citations will be manually retrieved. All identified papers from the selected databases will be assessed for eligibility by two independent reviewers.

\section{Inclusion and exclusion criteria}

This review will include observational studies conducted with cross-sectional, cohort and case-control study designs. Studies conducted in sub-Saharan African and published in English will be included to the review. To be included, the papers should reported on the status of MHM for female students, and included information about school absenteeism.. Articles will be included irrespective of their publication year. Commentaries, anonymous reports, letters and editorials will be excluded.

\section{Study screening}


Articles that are initially obtained from the selected databases will be exported to EndNote X9 library and exact duplicates will be removed. Then, the EndNote library will be shared between two reviewers (AG \& AGB) who will independently screen for articles by title and abstract. After finalization of abstract review, Cohen's kappa coefficient will be performed to judge for agreement between the reviewers. Acceptable agreement between the reviewers will be concluded if the Cohen's kappa coefficient obtained shows substantial agreement $(>0.60)$ (30). A full-text review of potentially relevant articles will be performed by the two reviewers ( $A G \& A G B$ ) independently. Any disagreements between the two reviewers will be solved through arbitration by a third reviewer (AKT).

\section{Data extraction}

Two reviewers will independently extract the data from the full text of the retained articles. Data will be extracted using an adapted Johanna Briggs Institute (JBI) data abstraction format (31) (Table 1). Study characteristics such as author and publication year, country, objective of the study, study design, study population, sampling method, sample size, data collection method, number/percent of female students who missed classes during menstruation, factors affecting school absenteeism will be extracted from all included articles. Missed data, if any, will be handled by contacting the corresponding author of the eligible articles, if required.

Table 1: Data extraction form, adapted from the JBI data extraction checklist 


\begin{tabular}{|l|}
\hline Study characteristics \\
\hline Authors and year \\
\hline Title of the study \\
\hline Objective of the study \\
\hline Study participants \\
\hline Study setting (country) \\
\hline Study design \\
\hline Sample size \\
\hline Sampling methods \\
\hline Data collection technique \\
\hline Data collection period \\
\hline Methods of analysis \\
\hline Ethical clearance provided from \\
\hline Quality score of the article \\
\hline Relevant findings (school absenteeism) \\
\hline Factors affected school attendance \\
\hline Author's conclusion \\
\hline
\end{tabular}

\section{Quality assessment and risk of bias}

The quality of the retained articles will be appraised independently by the two reviewers using an adapted Newcastle-Ottawa Scale (NOS) for cross-sectional studies (32). The NOS was reported to have a good inter-rater reliability and validity (33) and was effectively applied in observational studies (34). The tool gives a maximum score of nine with three categories of criteria. A maximum of four stars allotted for 'sample selection'; a maximum of two stars allotted for 'comparability'; and a maximum of three stars allotted for the 'outcome'. All studies will be included regardless of their quality rating. However, a subgroup analysis will be done to control their effect on the estimate.

Potential publication bias of the articles will be assessed using funnel plots and Egger's regression test (35). Before conducting the meta-analysis, arc-sine transformation of the prevalence estimates will be used to adjust the effect of studies with high prevalence of school absenteeism on the pooled estimate (36). Heterogeneity of the studies will be measured using the Higgins and colleagues method where $P$ statistic $>80 \%$ indicates substantial heterogeneity (37). 
The primary outcome for this review is school absenteeism during menstruation, defined as reported missing of at least one class during their menstruation. For quantitative findings, meta-analysis and meta-regression will be carried out to calculate the pooled level of school absenteeism and an OR of each associated factor. Effect size of the quantitative findings will be estimated and reported using randomeffect meta-analysis models $(38,39)$. Furthermore, a separate forest plot will be constructed for each subgroup analysis. The factors that are reported to affect school absenteeism will be qualitatively presented using a narrative synthesis if meta-analysis is not feasible due to the substantial heterogeneity of studies. Data entry and analysis will be done using Review Manager Software for windows.

\section{Discussions}

Although menstruation is a normal physiological process, it poses a huge physical and psychological burden on the majority of school girls $(40,41)$. Findings of several studies highlighted that pain experienced during menstruation (10), poor MHM in school (5), lack of water, sanitation and hygiene facilities in schools (6), and societal beliefs that restricts women during menstruation (15) were some of the factors that could affect school attendance during menstruation among female students. Moreover, in low income settings, adolescent girls lack appropriate awareness about menstruation and MHM (3). Systematic analysis of the existing evidence of school absenteeism due to menstruation is essential for designing appropriate interventions in school and beyond. Therefore, this systematic review and metaanalysis is expected to reveal a credible evidence through pooling the prevalence of school absenteeism and associated factors among female high school students in sub-Saharan Africa. This evidence will help in designing effective school based women health interventions to enhance school attendance and help girls student to go through their menstruation period as normal physiologic process (26).

\section{Abbreviations}

CINAHL Cumulative Index to Nursing and Allied Health Literature

JBI Johanna Briggs Institute

MOOSE Meta-analysis Of Observational Studies in Epidemiology

MHM Menstrual Hygiene Management

WASH Water, Sanitation and Hygiene

NOS Newcastle Ottawa Scale

PRISMA Preferred Reporting Items for Systematic Reviews and Meta-Analyses

\section{Declarations}

\section{Ethics approval and consent to participate}


Not applicable

\section{Consent for publication}

Not applicable

\section{Availability of data and materials}

All data extracted or analysed during this review will be included in the publication of this systematic review article.

\section{Competing interests}

The authors declare that they have no competing interests

\section{Funding}

This study has no specific funding organization.

\section{Authors' contributions}

AG developed the draft proposal under the supervision of MD and AKT. AGB, MD and AKT critically reviewed, provided substantive feedback and contributed to the intellectual content of this paper. All authors (AG, MD, AGB and AKT) made substantial contributions to the conception, conceptualization and manuscript preparation of this systematic review and meta-analysis protocol. All authors read and approved the final manuscript.

\section{Acknowledgements}

The authors would like to thank Haramaya University for supporting AG to attend her master education. We would also like to extend our thanks to library workers in Haramaya University College of Health and Medical Sciences for supplying us with necessary references without which this review could not be completed.

\section{Authors' information}

AG is a Master of Public Health student; she holds a bachelor of Nursing in clinical nurse, and is currently working in Ambo Referral Hospital, College of Medicine and Health Sciences, Ambo University, Ambo, Ethiopia. AGB (PhD) is currently working in the Research Centre for Generational Health and Aging, School of Medicine and Public Health, University of Newcastle, Australia. MD is an Asst. professor of public health and currently serves in Institutional Health Research Ethics Review Committee of Haramaya University, College of Health and Medical Sciences. AKT (PhD) is currently working as coordinator; school of postgraduate study in College of Health and Medical Sciences, Haramaya University, Harar, Ethiopia. 


\section{References}

1. Jogdand K, Yerpude P. A community based study on menstrual hygiene among adolescent girls. Indian journal of maternal and child health. 2011;13(3):1-6.

2. McPherson ME, Korfine L. Menstruation across time: menarche, menstrual attitudes, experiences, and behaviors. Women's Health Issues. 2004;14(6):193-200.

3. Oche M, Umar A, Gana G, Ango J. Menstrual health: the unmet needs of adolescent girls in Sokoto, Nigeria. Scientific Research and Essays. 2012;7(3):410-8.

4. Dasgupta A, Sarkar M. Menstrual hygiene: how hygienic is the adolescent girl? Indian journal of community medicine: official publication of Indian Association of Preventive \& Social Medicine. 2008;33(2):77.

5. Sommer M, Sahin M. Overcoming the taboo: advancing the global agenda for menstrual hygiene management for schoolgirls. Am J Public Health. 2013;103(9):1556-9.

6. Mason L, Nyothach E, Alexander K, Odhiambo FO, Eleveld A, Vulule J, et al. 'We keep it secret so no one should know'-A qualitative study to explore young schoolgirls attitudes and experiences with menstruation in rural Western Kenya. PloS one. 2013;8(11):e79132.

7. Bodat S, Ghate MM, Majumdar JR. School absenteeism during menstruation among rural adolescent girls in Pune. Natl J Community Med. 2013;4(2):212-6.

8. Bobhate PS, Shrivastava SR. A cross sectional study of knowledge and practices about reproductive health among female adolescents in an urban slum of Mumbai. 2011.

9. Adhikari P, Kadel B, Dhungel S, Mandal A. Knowledge and practice regarding menstrual hygiene in rural adolescent girls of Nepal. Kathmandu University medical journal (KUMJ). 2007;5(3):382-6.

10. Muluneh AA, seyuom Nigussie T, Gebreslasie KZ, Anteneh KT, Kassa ZY. Prevalence and associated factors of dysmenorrhea among secondary and preparatory school students in Debremarkos town, North-West Ethiopia. BMC Womens Health. 2018;18(1):57.

11. Adinma ED, Adinma J. Perceptions and practices on menstruation amongst Nigerian secondary school girls. Afr J Reprod Health. 2008;12(1):74-83.

12. Ortiz MI, Rangel-Flores E, Carrillo-Alarcón LC, Veras-Godoy HA. Prevalence and impact of primary dysmenorrhea among Mexican high school students. International Journal of Gynecology \& Obstetrics. 2009;107(3):240-3.

13. Nooh AM. Menstrual disorders among Zagazig university students, Zagazig, Egypt. Middle East Fertility Society Journal. 2015;20(3):198-203.

14. Shah M, Monga A, Patel S, Shah M, Bakshi H. A study of prevalence of primary dysmenorrhea in young students-A cross-sectional study. Healthline. 2013;4(2):30-4.

15. Thakur H, Aronsson A, Bansode S, Stalsby Lundborg C, Dalvie S, Faxelid E. Knowledge, practices, and restrictions related to menstruation among young women from low socioeconomic community in Mumbai, India. Frontiers in public health. 2014;2:72. 
16. Enoch A, Nadutey A, Afful BF, Anokye R. Menstrual Hygiene Management: Challenges and Coping Strategies for Adolescents with Disabilities in the Kumasi Metro of Ghana. Disability, CBR \& Inclusive Development. 2020;31(2).

17. Mahon T, Fernandes M. Menstrual hygiene in South Asia: a neglected issue for WASH (water, sanitation and hygiene) programmes. Gender \& Development. 2010;18(1):99-113.

18. Oster E, Thornton R. Menstruation, sanitary products, and school attendance: Evidence from a randomized evaluation. American Economic Journal: Applied Economics. 2011;3(1):91-100.

19. Tegegne TK, Sisay MM. Menstrual hygiene management and school absenteeism among female adolescent students in Northeast Ethiopia. BMC Public Health 2014;14(1118).

20. McMahon SA, Winch PJ, Caruso BA, Obure AF, Ogutu EA, Ochari IA, et al. 'The girl with her period is the one to hang her head'Reflections on menstrual management among schoolgirls in rural Kenya. BMC international health and human rights. 2011;11(1):7.

21. El Gilany A, Badawi K, El Fedawy S. Epidemiology of dysmenorrhoea among adolescent students in Mansoura, Egypt. 2005.

22. Titilayo A, Agunbiade O, Banjo O, Lawani A. Menstrual discomfort and its influence on daily academic activities and psychosocial relationship among undergraduate female students in Nigeria. Tanzan J Health Res. 2009;11(4).

23. Esimai O, Esan GO. Awareness of menstrual abnormality amongst college students in urban area of Ile-Ife, Osun state, Nigeria. Indian journal of community medicine: official publication of Indian Association of Preventive \& Social Medicine. 2010;35(1):63.

24. Santina T, Wehbe N, Ziade F. Exploring dysmenorrhoea and menstrual experiences among Lebanese female adolescents. Eastern Mediterranean Health Journal. 2012;18(8):857-63.

25. Fehr A. Stress, menstruation and school attendance: effects of water access amongadolescent girls in South Gondar, Ethiopia. Atlanta, GA: Emory University. 2011.

26. Bartram J, Lewis K, Lenton R, Wright A. Focusing on improved water and sanitation for health. The Lancet. 2005;365(9461):810-2.

27. Miiro G, Rutakumwa R, Nakiyingi-Miiro J, Nakuya K, Musoke S, Namakula J, et al. Menstrual health and school absenteeism among adolescent girls in Uganda (MENISCUS): a feasibility study. BMC Womens Health. 2018;18(1):4.

28. Page MJ, Moher D. Evaluations of the uptake and impact of the Preferred Reporting Items for Systematic reviews and Meta-Analyses (PRISMA) Statement and extensions: a scoping review. Syst. 2017:6(1):263.

29. Stroup DF, Berlin JA, Morton SC, Olkin I, Williamson GD, Rennie D, et al. Meta-analysis of observational studies in epidemiology: a proposal for reporting. Jama. 2000;283(15):2008-12.

30. Viera AJ, Garrett JM. Understanding interobserver agreement: the kappa statistic. Fam med. 2005;37(5):360-3. 
31. Joanna Briggs Institute. JBI Manual for evidence synthesis: Data Extraction Form for Review for Systematic Reviews and Research Syntheses. 2014.

32. Peterson J, Welch V, Losos M, Tugwell P. The Newcastle-Ottawa scale (NOS) for assessing the quality of nonrandomised studies in meta-analyses. Ottawa: Ottawa Hospital Research Institute. 2011.

33. Hartling L, Hamm M, Milne A, Vandermeer B, Santaguida PL, Ansari M, et al. Validity and inter-rater reliability testing of quality assessment instruments. 2012.

34. Habtewold TD, Islam MA, Sharew NT, Mohammed SH, Birhanu MM, Tegegne BS. SystEmatic review and meta-aNAlysis of infanT and young child feeding Practices (ENAT-P) in Ethiopia: protocol. BMJ open. 2017;7(8):e017437.

35. Egger M, Smith GD, Schneider M, Minder C. Bias in meta-analysis detected by a simple, graphical test. Bmj. 1997;315(7109):629-34.

36. Barendregt JJ, Doi SA, Lee YY, Norman RE, Vos T. Meta-analysis of prevalence. J Epidemiol Community Health. 2013;67(11):974-8.

37. Higgins JP, Thompson SG. Quantifying heterogeneity in a meta-analysis. Statistics in medicine. 2002;21(11):1539-58.

38. Borenstein M, Hedges LV, Higgins JP, Rothstein HR. A basic introduction to fixed-effect and randomeffects models for meta-analysis. Research synthesis methods. 2010;1(2):97-111.

39. DerSimonian R, Laird N. Meta-analysis in clinical trials revisited. Contemp Clin Trials. 2015;45:13945.

40. Shanbhag D, Shilpa R, D'Souza N, Josephine P, Singh J, Goud B. Perceptions regarding menstruation and practices during menstrual cycles among high school going adolescent girls in resource limited settings around Bangalore city, Karnataka, India. International Journal of Collaborative Research on Internal Medicine \& Public Health. 2012;4(7):1353.

41. Davis J, Macintyre A, Odagiri M, Suriastini W, Cordova A, Huggett C, et al. Menstrual hygiene management and school absenteeism among adolescent students in Indonesia: evidence from a cross-sectional school-based survey. Tropical Medicine \& International Health. 2018;23(12):1350-63.

\section{Figures}




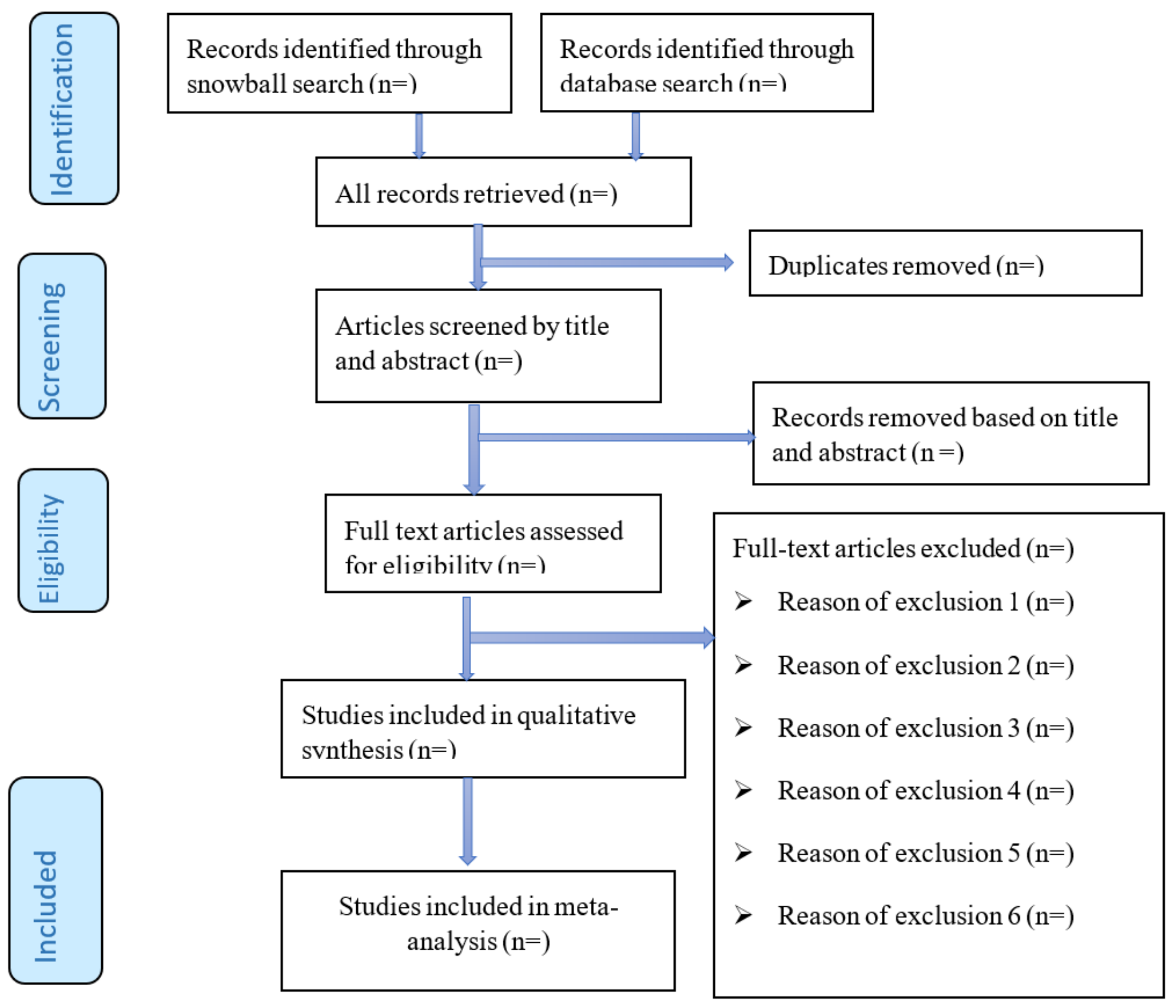

Figure 1

PRISMA flow diagram showing the selection of the included articles

\section{Supplementary Files}

This is a list of supplementary files associated with this preprint. Click to download.

- MEDLINEsearchsample.docx 